\title{
Mikko Huotari/Jan Gaspers/Thomas Eder/Helena Lagarda/Sabine Mokry: China's Emergence as a Global Security Actor. Strategies for Europe. Berlin: Mercator Institute for China Studies (MERICS), Juli 2017.
}

Besprochen von Dr. Till Florian Tömmel, Jiaotong Universität, Shanghai; E-Mail: till.toemmel@unibw.de

https://doi.org/10.1515/sirius-2018-4020

China ist dabei, sich zu einem weltpolitischen Akteur mit umfassenden Ambitionen zu entwickeln - so die Aussage des hier besprochenen Papiers aus dem Hause MERICS in Berlin, einer führenden Adresse für Fragen der Chinapolitik. Die Autoren analysieren Entwicklungslinien, die seit der Übernahme der höchsten Staats- und Parteiämter durch Xi Jinping 2012/13 deutlich auf eine grundlegend veränderte Grand Strategy Chinas verweisen. Um den Fortgang dieser Entwicklungen auf den Zeithorizont 2022 hin zu prognostizieren, stützt sich die Studie auf eine anonymisierte Delphi-Befragung von 35 Fachleuten. Das Ergebnis lautet: Peking werde zukünftig auf vielen Feldern mitentscheiden, auf denen es zuvor kein oder nur wenig Profil gezeigt hat. Grundsätzlich müssten sich EU-Staaten auf eine Infragestellung ihrer Präferenzen einstellen. In bestimmten Bereichen sei aber Kooperation mit Chinas Führung möglich, etwa bei UN-Friedensmissionen. 
Die Volksrepublik und ihre Akteure prägten die internationale Politik in vier idealtypisch zu verstehenden Rollen: Als Diplomat, als Soldat, als Händler sowie als Gestalter (shaper). Als „Diplomat“ sei Peking erfolgreich darin, Partner für seine Sicherheits- und Verteidigungspolitik zu gewinnen. Hierfür stehe ein breit gefächertes Instrumentarium zur Verfügung, das von der sicherheitspolitischen Nutzung ökonomischer Ressourcen über bilaterale Auslieferungsabkommen bis zur Militärhilfe für afrikanische Staaten reiche. Die Volksbefreiungsarmee (VBA) baue ihre Militär-zu-Militär-Diplomatie aus. Diese beschränke sich längst nicht mehr auf die wenigen traditionellen Sicherheitspartner Chinas: In den letzten Jahren wurden Beziehungen zu NATO-Armeen hergestellt, u.a. zur Bundeswehr. Deutsche Sanitätssoldaten haben im Oktober 2016 erstmals eine gemeinsame Übung mit der VBA abgehalten.

Auch in der Rolle des „Soldaten“ zeige China zunehmend globale Dimensionen und Ambitionen. Dabei folge die chinesische Militärpolitik der bereits bestehenden wirtschaftlichen Globalpräsenz Chinas. Hatte noch das amtliche Weißbuch von 2010 der VBA einen recht engen Aufgabenbereich zugewiesen, so erweiterte das nachfolgende Weißbuch von 2015 ihren strategischen und operativen Radius. Erstmals wurde der militärische Schutz chinesischer Interessen im Ausland explizit festgeschrieben und die Doktrin der „Nicht-Einmischung in innere Angelegenheiten" aufgeweicht. Eine noch deutlichere kommunikative Abkehr von der überkommenen Nichtinterferenz stellt das Anti-Terrorismus-Gesetz von 2015 dar, das Chinas Militär und Sicherheitskräften unter bestimmten Bedingungen zur Terrorismusbekämpfung im Ausland ermächtigt. In diesem Zusammenhang werde das gigantische Infrastrukturvorhaben One Belt One Road erhebliche sicherheitspolitische Begleitmaßnahmen erfordern, da es durch instabile Räume in Zentral- und Südasien führen soll. Neben Afghanistan und Pakistan muss auch Chinas Region Xinjiang zur „Gefahrenzzone“ gerechnet werden. Besondere Sorge haben die Behörden vor nach Xinjiang zurückkehrenden Uiguren, die im Irak und in Syrien in sunnitischen Extremistengruppen gekämpft haben.

Chinas See- und Luftstreitkräfte werden aufwändig modernisiert, u. a. durch neue Flugzeugträger, Fregatten und nukleargetriebene U-Boote sowie durch Transportmaschinen und Kampfflugzeuge mit Tarnkappentechnik. Der Studie zufolge werde die VBA bis 2022 Fähigkeiten zur Machtprojektion entwickelt haben, die ihr mehrere kleinere Operationen zur gleichen Zeit erlauben - auch weit entfernt von der Heimatregion. Schon 2011 evakuierten chinesische Truppen rund 35.000 Chinesen aus Libyen;
2015 dann über 800 Personen aus dem Jemen, darunter 225 Nichtchinesen. 2017 eröffnete die Marine ihren ersten Auslandsstützpunkt in Dschibuti. Die Autoren gehen davon aus, dass der maritime Teil der „neuen Seidenstraße" den Aufbau eines Logistiknetzwerkes mit weiteren Stützpunkten erfordern wird. Zudem erschließe sich China neue Räume: Der Aufbau des Satellitennavigationssystems Běidǒu soll bis 2020 fertiggestellt werden. Laut Weißbuch von 2015 sind der erdnahe Weltraum und der Cyberspace die „neuen Kommandohöhen im strategischen Wettbewerb“ (zhànlüè jìngzhēng xīn de zhìgāodiăn).

Seine Position als Handelsmacht (trader-Rolle) nutzt China effektiv für die Verfolgung geostrategischer Interessen. Ausländischen Regierungen setzt die Staats- und Parteiführung durch Handelsbeziehungen, Wirtschaftshilfen, Direktinvestitionen, gezielte Unternehmenskäufe sowie das Gewähren oder Versagen von Marktzugang starke Anreize, sich den politischen Präferenzen Chinas anzupassen. Oft brauche es dazu keine explizite Bitte oder Drohung. Besonders markant werde der ökonomisch-strategische Nexus bei der chinesischen Rüstungsindustrie sichtbar. Für verschiedene Länder in Süd- und Südostasien ist China mittlerweile der führende Waffenlieferant, darunter für Pakistan und Bangladesch. Besonders dynamisch entwickelten sich die Rüstungsexporte nach Afrika, wo mittlerweile zwei Drittel der Staaten Waffen aus dem Reich der Mitte führen. Auch wenn die Volksrepublik noch weit hinter den USA und Russland liegt, prognostizieren die Autoren bis 2022 einen Anteil von etwa zehn Prozent am globalen Waffenhandel. Wahrscheinlich würden sich einige weniger komplizierte Waffensysteme zu „Verkaufsschlagern“ entwickeln, etwa Drohnen und bestimmte Raketentypen.

Die schon erwähnte Belt and Road Initiative (BRI) verbinde auf organische Weise wirtschaftliche und strategische Ziele miteinander. Neben wachstums-, export-, handels-, energie- und währungspolitischen Motiven werde die Absicht erkennbar, eine asymmetrische Interdependenz zu schaffen. Einer Publikation der Pekinger Akademie für Militärwissenschaften von 2016 zufolge solle die BRI eine Zugwirkung für ein stärkeres militärischstrategisches Profil der Volksrepublik entfalten. Der kanadisch-indische Politikwissenschaftler Amitav Acharya hat in diesem Zusammenhang darauf hingewiesen, dass die „neue Seidenstraße“ ein wirtschaftliches Äquivalent zum amerikanischen Netzwerk aus Militärbündnissen darstelle. Elemente klassischer Machtpolitik weise die BRI insofern auf, als durch die entstehende chinesische Infrastruktur der Einfluss Indiens in Südasien drastisch beschnitten würde. 
Die letzte diskutierte Rolle sieht Chinas als „Gestalter" der internationalen Politik. Es gehe dabei v.a. um das Bereitstellen von Modellen und Konzepten über das eigene Land hinaus. Die Studie diagnostiziert, dass sich China seit dem Machtwechsel von 2012/13 nicht mehr mit der lang gepflegten Zurückhaltung nach außen zugunsten innerer Weitentwicklung zufriedengebe, sondern daran arbeite, seine staats- und souveränitätsbetonte Sicherheitspolitik zu externalisieren, etwa in den Vereinten Nationen oder in Regionalformaten wie der Shanghai-Organisation. Xi äußerte im März 2017 programmatisch, die Volksrepublik könne die Staatengemeinschaft in Fragen der internationalen Ordnung und internationalen Sicherheit „anführen“ (y̌ndăo). Der in der Gegenwart wahrgenommene Niedergang westlicher Ordnungsmodelle bietet einen dazu passenden Hintergrund. Ein Politikfeld, in dem Peking sich um die globale Verbreitung des eigenen Ansatzes bemühe, sei die Regulierung und Kontrolle des Internets. Die Autoren erwarten bei diesem Thema zukünftigen Dissens mit den Präferenzen liberaler westlicher Regierungen.

Die Studie schließt mit einer langen Reihe von Empfehlungen an die Adresse europäischer Regierungen, die nahezu ausschließlich der soft security zuzuordnen sind. Dazu gehört der Ratschlag, im Rahmen der NATO einen transatlantischen Dialog über die strategische Bedeutung der wachsenden VBA-Präsenz an den Grenzen Europas zu führen, der perspektivisch auch Indien und Japan in die Konsultationen einbeziehen solle. Ob die europäische Asienpolitik von chinesischer Seite ernstgenommen wird, dürfte wesentlich vom Willen und der Fähigkeit Deutschlands, Frankreichs und Großbritanniens (unabhängig vom Status seiner EU-Zugehörigkeit) abhängen, ein deutliches Profil zu zeigen.

https://www.merics.org/de/papers-on-china/chinasemergence-global-security-actor 